\title{
Panorama general de las visitas en las unidades de cuidado intensivo'
}

\author{
Luisa Fernanda Achury Beltrán²
}

doi:10.11144/Javeriana.IE16-1.pgvu

Cómo citar: Achury Beltrán LF. Panorama general de las visitas en las unidades de cuidado intensivo. Investig Enferm. Imagen Desarr. 2014;16(1): 61-71. doi:10.11144/Javeriana.IE16-1.pgvu

1. Artículo de revisión. Recibido: 24 de septiembre de 2013. Revisado: 21 de enero de 2014. Aprobado: 27 de enero de 2014.

2. Enfermera especialista en Cuidado Crítico, Pontificia Universidad Javeriana, Bogotá, Colombia. Profesora instructora, Facultad de Enfermeria, Pontificia Universidad Javeriana. Correo electrónico: achuryl@javeriana.edu.co. 


\section{Resumen}

Las unidades de cuidado intensivo (UCI) se conciben como espacios cerrados, debido a la complejidad de los pacientes que allí se manejan. Por esta razón, las visitas se han convertido en un aspecto de gran importancia, ya que influyen en la satisfacción de las necesidades de los pacientes, sus familias y las creencias del personal de salud. Hablar del panorama de las visitas en las UCI, implica retomar su origen y evolución, teniendo en cuenta que las nociones acerca de la gravedad de los pacientes alli hospitalizados y las creencias de quienes los atienden han marcado un punto importante en la restricción histórica de ellas. De igual manera, es necesario mencionar las ventajas de una politica liberalizada, conocer la perspectiva general de las visitas en las UCI y el papel que desempeña el profesional de enfermería en este aspecto. Este artículo pretende motivar en las(os) enfermeras(os) una participación más activa en la toma de decisiones sobre las politicas de visitas en la UCI, a partir del conocimiento de su panorama y los efectos que tienen las políticas flexibles en la salud del paciente y la estabilidad de la familia.

Palabras clave: enfermería, cuidados intensivos, visitas a pacientes.

Palabras clave descriptor: enfermeria, cuidados intensivos, visitas a pacientes.

\section{Overview of visits in intensive care units}

\section{Abstract}

Intensive care units (ICU) are conceived as closed spaces, due to the complexity of patients which are handled therein. For this reason, visits have become an issue of great importance, since they influence the satisfaction of the patients' needs, their families and beliefs of health personnel. Talking about the overview of visits in the ICU involves going back to their origin and evolution, considering that the notions about the severity of the patients which are hospitalized there and the beliefs of their caregivers have marked an important point in the historical restriction of those visits. Similarly, it is necessary to mention the benefits of a liberalized policy, get to know the general perspective of visitors in the ICU and the role of the nursing staff in this regard. This article seeks to motivate the nurses to have a more active participation in the decision-making on policies regarding the ICU visits, departing from their knowledge of their outlook and the effects flexible policies have on the patients' health and family stability.

Keywords: nursing, intensive care, patient visits.

Key words plus: nursing, intensive care, visits to patients. 


\section{Panorama geral de visita na unidade de tratamento intensivo}

\section{Resumo}

As unidades de tratamento intensivo (UTI) são concebidas como espaços fechados devido à complexidade dos pacientes ali tratados. Por isso, a visita tem se tornado um aspecto de grande importância já que influi na satisfação das necessidades do paciente, sua família e as crenças do pessoal de saúde. Falar do panorama da visita na UTI implica retomar a origem e evolução, levando em conta que as noções acerca da acuidade dos pacientes internados lá e as crenças de quem cuida marcaram um ponto importante na restrição histórica delas. De igual maneira, é preciso mencionar as vantagens de uma política liberalizada, conhecer a perspectiva geral da visita na UTI e o papel desenvolvido pelo profissional de enfermagem neste aspecto. Este artigo pretende motivar as(os) enfermeiras(os) para uma participação mais ativa na tomada de decisões sobre as políticas de visita na UCI a partir do conhecimento do seu panorama e os efeitos que as políticas flexiveis têm na saúde do paciente e a estabilidade da família.

Palavras-chave: enfermagem, tratamento intensivo, visitas a pacientes.

Palavras chave descriptor: enfermagem, terapia intensiva, visitas a pacientes. 


\section{Las visitas en las unidades de cuidado intensivo y los factores que las influencian}

Las unidades de cuidado intensivo (UCI) tienen su primer antecedente en el siglo XIX, cuando Florence Nightingale agrupaba a los heridos de la guerra de Crimea según su gravedad, poniéndolos a cargo de las enfermeras con mayor experiencia y conocimientos (1). A partir de ese momento, las UCI evolucionaron de manera progresiva, centradas en el manejo de la persona en estado crítico y bajo los más altos estándares de calidad, tanto en recurso humano como en recursos físicos y tecnológicos, con el objetivo de favorecer la recuperación de los pacientes (2); sin embargo, su condición crítica llevó a pensar que las visitas podrian llegar a tener efectos deletéreos, lo que les imprimió a las UCI el carácter de espacios cerrados y, a su vez, condicionó la aparición del perfil restrictivo que todavía permea las politicas de visitas (3).

En 1988, Stockdale y Hughes, citados en Martínez (1), realizaron un estudio con enfermeras de $197 \mathrm{UCI}$, en el cual encontraron que la mayoría de dichas unidades limitaba el número de las visitas, el tiempo de cada una de ellas, la edad mínima de los visitantes y la cantidad de acompañantes permitida, de manera que solo hasta 1992, Hopping y colaboradores, citados en Martínez (1), encontraron en sus estudios politicas de visitas significativamente más liberales, las cuales eran características de los hospitales universitarios.

No obstante, a pesar de las modificaciones el carácter poco flexible de las políticas de visitas en las UCI, está fuertemente permeado por las regulaciones que han existido en torno a este aspecto y las percepciones de quienes cuidan al paciente en estado crítico.

En términos de las regulaciones, en 1962, el servicio de salud pública de Estados Unidos estableció unas recomendaciones que sugerían visitas limitadas a cinco minutos cada hora y la provisión de un área de espera cercana a la unidad; en 1965, la revisión a estas recomendaciones sugirió unas modificaciones a las restricciones, basadas en la condición del paciente y en la habilidad del personal para manejar a los visitantes $(4,5)$, aspectos que todavía influyen de manera significativa en los actuales lineamientos, pues a partir de la publicación de estas guías, el cambio hacia politicas de visitas menos restrictivas ha sido un proceso realmente lento (6).

Sin embargo, las leyes y normativas no son el único factor que toma parte en las decisiones respecto a las políticas de visitas en la UCI, pues es evidente que las creencias y las actitudes del profesional de enfermería desempeñan un papel esencial en el proceso desde que les fueron otorgadas facultades para decidir acerca de su liberalización o no.

Diversos estudios, han revelado que las percepciones del personal de salud, especialmente de las enfermeras, subestiman las propiedades benéficas de las visitas abiertas y, así mismo, maximizan las creencias sobre sus efectos deletéreos, sin la existencia de evidencia científica que lo sustente, de modo que los profesionales de enfermería no consideran que 
proporcionar un apoyo para los cuidadores (7). De forma similar, creen que las visitas flexibles interfieren con la planeación del cuidado, incrementan el tiempo que el enfermero utiliza brindando información a los familiares, obstaculizan con el cuidado directo de enfermería, favorecen el agotamiento de los pacientes y familiares, interfieren con el humor de las enfermeras, incrementan el estrés psicológico del paciente, hacen que los profesionales se sientan controlados, generan descompensación hemodinámica en los pacientes e infringen su privacidad e incrementan el riesgo de infección (7-14), por lo que restringen las visitas e implementan barreras para su flexibilización (8).

Es evidente que las enfermeras de las UCI son muy escépticas ante la apertura de las visitas, lo cual se constituye en una barrera importante cuando los hospitales desean liberalizar sus politicas de acuerdo con la nueva evidencia (7). Según Zaforteza y colaboradores (15), este aspecto puede estar relacionado con el desconocimiento por parte de los profesionales acerca de las necesidades de la familia y de los efectos benéficos de una politica no restrictiva de visitas, con la cultura dominante de la UCI y con la carencia de herramientas por parte de los profesionales de enfermería para atender la angustia de los familiares.

Sin embargo, a pesar de la influencia de las percepciones de los profesionales y las regulaciones, es necesario resaltar los efectos benéficos de una politica liberalizada de visitas en la UCI para los actores que influyen en el proceso: el paciente, el familiar y el equipo de salud.

\section{Efectos benéficos de la flexibilización de las políticas de visitas en la unidad de cuidado intensivo}

La literatura sobre el tema avala los cambios en las políticas de visitas en la UCI hacia unos perfiles más flexibles y liberales que permitan a los familiares un mayor contacto con el paciente crítico, la obtención de mayor información y, en general, un incremento en la satisfacción de las necesidades tanto del paciente como de su familia (16-20).

Para el paciente, una politica menos restrictiva repercute positivamente en un incremento en la satisfacción de las necesidades; una reducción de la ansiedad, la agitación, la confusión y el estrés; una disminución de las complicaciones cardiovasculares asociada con un descenso de la ansiedad y un mejor control del perfil hormonal de estrés; así como un incremento en la sensación de aprecio y seguridad percibida por el paciente, ya que la familia le provee un apoyo emocional, lo que finalmente trasciende en la reducción del tiempo de estancia en la UCI (21-26).

Por otra parte, la liberalización de las visitas incrementa la satisfacción de las necesidades familiares, dentro de las cuales la de proximidad, seguridad e información son prioritarias; así mismo, se considera que reduce el estrés y la ansiedad en los familiares, promueve una mejor comunicación tanto con el paciente como con el personal de salud, asegura una mayor información como parte de la satisfacción de las necesidades familiares y permite la participación familiar en el cuidado del paciente crítico (27-30). 
Finalmente, a pesar de las percepciones negativas, algunos estudios demuestran que las enfermeras también perciben aspectos positivos derivados de una política de visitas menos restrictiva, por lo que manifiestan aumento de la satisfacción laboral (debido a la retroalimentación que se produce con el familiar), incremento de las oportunidades para la educación del paciente y la familia, mejoría en el proceso de comunicación, avance en la entrega de cuidado de enfermería (por la información obtenida del paciente en el contacto con sus familiares) y mejoría en la relación entre el personal y la familia (31-34).

Con base en los beneficios reportados por la literatura, es imprescindible conocer el comportamiento general de las visitas en las UCI, determinar necesidades de cambios en las actuales politicas y establecer el papel que debe desempeñar el profesional de enfermería en este aspecto.

\section{Comportamiento general de las visitas en las unidades de cuidado intensivo}

En el ámbito internacional, el panorama de las visitas en las UCI de Estados Unidos parece ser bastante positivo, pues el estudio de Khirchhoff y Dahl (35) reveló que el $44 \%$ de las UCI mantiene políticas abiertas de horario; el $31 \%$ no ejerce restricciones, excepto durante las rondas de enfermería o los cambios de turno, y un $14 \%$ de las unidades mantiene politicas abiertas de forma continua, independientemente de las actividades del personal de salud.

Sin embargo, en Europa el panorama no parece ser tan flexible, pues los estudios muestran que en Italia solo el $1 \%$ de las UCI maneja la liberalización de visitas. El estudio de Gianni, Miccinesi y Leoncino (36) encontró que las restricciones se relacionan en un $92 \%$ con el número de visitantes permitidos, en un $17 \%$ con el tipo de acompañante y en un $69 \%$ con el ingreso de los niños a las unidades. A pesar de esto, el $33 \%$ de las políticas de visitas se encuentra en procesos de revisión.

Por otra parte, en Francia el estudio de Quinio y colaboradores (37) reflejó que solo el $3 \%$ de las UCI maneja politicas abiertas de visitas y las restricciones de las unidades restantes se relacionan en un $95 \%$ con el número de visitantes, en un $60 \%$ con el tipo de acompañantes (pues permite solo el ingreso de los familiares cercanos) y en un $11 \%$ con el ingreso de los niños.

En España (38), el $63 \%$ de las UCI solo tienen permitidas visitas dos veces al día, el 58\% tiene establecidas visitas de media hora y también realizan restricciones en cuanto al número de familiares a los que se les permite el ingreso.

Un estudio realizado en Holanda reveló que ninguna de las UCI tenía visitas abiertas, las cuales están definidas como una política que no impone ninguna restricción en el tiempo de visita, duración de las visitas o número de visitantes, de forma tal que el 85,7\% de las unidades contaba de los efectos benéficos de las visitas sin restricciones (39). 
En el Reino Unido (40), un estudio demostró que el 80,1\% de las unidades siguen imponiendo restricciones a las visitas, pese a las amplias variaciones a las instalaciones que se encuentran a disposición de los familiares de los pacientes. Se encontró que aun cuando la mayoría de las unidades restringe el número de visitantes, solo el 3,8\% limita el ingreso de los niños.

Un estudio realizado específicamente en Inglaterra demostró que en este país el $32 \%$ de las unidades mantiene políticas abiertas y las restricciones solo se limitan a la edad de los visitantes, en un $58 \%$, y al número de acompañantes, en un $85 \%$ (41), aspectos que parecen ser razonables, de acuerdo con la organización estructural y funcional de las UCI.

Por otro lado, Suecia es el país del continente europeo que maneja el mayor porcentaje de politicas abiertas, pues en el $70 \%$ de sus UCI no se establece ningún tipo de restricción en las horas de visitas (42).

Un estudio realizado en Irán demostró que el 90,1\% de las UCI maneja políticas de visitas restrictivas: número de visitas permitidas a la semana, cantidad de visitantes simultáneos y visitas de menores de edad. Además, llama la atención que el 39\% de las unidades no tiene permitidas las visitas y el 17\% contempla visitas de menos de una hora. E1 93\% restringe la entrada de los menores de edad y el 56,3\% de las visitas está a cargo de las autoridades del personal, mas no del personal de salud (43).

Finalmente, en cuanto al panorama colombiano, aproximadamente el $90 \%$ de las UCI tiene políticas restrictivas, las cuales están dadas por periodos de visitas que oscilan entre quince minutos y dos horas, con una frecuencia de dos veces al día. Adicionalmente, algunas de las unidades manejan una relación tiempo-visitante que permite a cada uno de los familiares el ingreso por un periodo de quince minutos y, así, una cantidad máxima de cuatro visitantes en una hora. En otras unidades las restricciones están dadas por el número de personas, pues se permite solo el ingreso de un familiar. Otras politicas, entre tanto, establecen que solo debe ser permitido el ingreso de los parientes más cercanos.

Con base en el panorama de visitas y los ya reconocidos efectos derivados de las politicas liberalizadas, es indispensable que los profesionales de enfermería asuman un papel activo que promueva la flexibilización de las visitas y, con ello, se maximicen los desenlaces positivos resultantes de la interacción entre el profesional, el paciente y su familia. Tal resultado puede llegar a conseguirse incrementando el conocimiento de las enfermeras en aspectos como las necesidades familiares, los efectos benéficos de las políticas liberalizadas de visitas, el impacto de esta medida tanto en la satisfacción de las necesidades familiares $(44,45)$ como en la percepción de la calidad del cuidado y en herramientas que les permitan a los profesionales una mejor atención al familiar del paciente crítico.

De forma adicional, el profesional de enfermería debe ejercer el liderazgo clínico (46), el cual le permitirá equilibrar las necesidades de los pacientes, los familiares y el profesional de salud y, a la vez, empoderarse de su rol en el establecimiento de los lineamientos y el direccionamiento de las politicas de visitas en la UCI, como parte de la apertura al proceso de vinculación familiar. 
Así mismo, las enfermeras deben fortalecer sus habilidades comunicativas (47) y desarrollar estrategias en que faciliten el acompañamiento al familiar del paciente crítico (48), pues el ingreso de un individuo a la UCI es considerado una situación estresante, capaz de desestabilizar el núcleo familiar y sus relaciones. Zaforteza (49) considera que las enfermeras requieren formación más amplia en el campo de la salud mental, para poder brindar cuidados que den respuesta a las necesidades de afrontamiento de los familiares de pacientes criticamente enfermos y a las situaciones de extrema tensión (50), lo que finalmente repercutirá en la satisfacción de los familiares y en el incremento de la percepción de la calidad del cuidado, aspectos fundamentales cuando se cuida de forma integral al paciente críticamente enfermo.

\section{Conclusiones}

- Existe suficiente evidencia acerca de los beneficios que traen consigo las politicas liberalizadas de visitas en la UCI tanto para el paciente como para la familia y el personal de salud, en términos de reducción de complicaciones, disminución de la ansiedad, aumento de la satisfacción e incremento en la percepción de la calidad del cuidado; no obstante, la mayoría de los estudios informa restricción de las visitas, las cuales se relacionan con el tiempo, la cantidad de familiares que ingresan y el ingreso de los niños.

- La restricción en la política de visitas en la UCI guarda relación en gran medida con la normatividad que se ha establecido desde su creación, la cual le ha impuesto un carácter cerrado y restrictivo; sin embargo, la percepción de los profesionales también cumple un papel importante, pues las creencias que giran en torno al estado de salud del paciente, la necesidad de descanso y los posibles efectos deletéreos de las visitas han dificultado su proceso de flexibilización.

- $\quad$ El profesional de enfermería desempeña un rol importante en el proceso de flexibilización de las visitas en la UCI, ya que puede participar de forma proactiva en el establecimiento de directrices y lineamientos con base en la evidencia científica. Para ello es preciso que se apropie del conocimiento referente a las visitas y, con ello, del desarrollo y fortalecimiento de herramientas y estrategias que le faciliten la comunicación con las familias y el apoyo en los momentos de crisis.

\section{Referencias}

1. Martínez G. Cuidados intensivos: necesidad de cuidados intermedios. Enferm Intensiva. 2002;13(3):113-24.

2. Weil $\mathrm{MH}$, Tang W. From intensive care to critical care medicine: a historical perspective. Am J Respir Crit Care Med. 2011;183:1451-3.

3. Cullen L, Titler M, Drahozal R. Family and pet visitation in the critical care unit. Crit Care Nurs. 1999;19(3):84-7. 
4. Hamner JB. Visitation policies in the ICU: A time for change. Crit Care Nurs. 1990;10(1):48-53.

5. Hopping B, Sickbert S, Ruth J. A study of factors associated with CCU visiting policies. Crit Care Nurs. 1992;12(2):8-15.

6. Farrell M, Hunt D, Schwartz-Barcott D. Visiting hours in the ICU: Finding the balance among patient, visitor and staff needs. Nursing Forum. 2005;40(1):18-28.

7. Berti D, Ferdinande P, Moons P. Beliefs and attitudes of intensive care nurses toward visits and open visiting policy. Intensive Care Med. 2007;33(6):1060-5.

8. Marco L, Bermejillo I, Garayalde N, Sarrate I, Margall M, Asiain M. Intensive care nurses' beliefs and attitudes towards the effect of open visiting on patients, family and nurses. Nurs Crit Care. 2006;11(1):33-41.

9. Karabacak U, Senturan L, Ozdilek S, Simsek A, Karateke Y, Ertekin $\mathrm{C}$, et al. The impact of visits on vital signs of the patients in surgical intensive care unit: a pilot study. Ulus Travma Acil Cerrahi Derg. 2012;18(1):18-22.

10. Biancofiore G, Bindi L, Barsotti E, Menichini S, Baldini S. Open intensive care units: a regional survey about the beliefs and attitudes of healthcare professionals. Minerva Anestesiol. 2010;76(2):93-99.

11. Azzi R, Bambi S. Open intensive care units: a feasible option? The opinions of patients, relatives and health care workers. Assist Inferm Ric. 2009;28(2):89-95.

12. Berwick DM, Kotagal M. Restricted visiting hours in ICUs: time to change. JAMA. 2004;292:736-7.

13. Adams S, Herrera A, Miller L, Soto R. Visitation in the intensive care unit: impact on infection prevention and control. Crit Care Nurs Q. 2011;34:3-10.

14. Tang CS, Chung FF, Lin MC, Wan GH. Impact of patient visiting activities on indoor climate in a medical intensive care unit: a 1-year longitudinal study. Am J Infect Control. 2009;37(3):183-8.

15. Zaforteza C, García A, Quintana R, Sanchez C, Abadía E, Albert J. Abrir la unidad de cuidados intensivos a los familiares: ¿qué opinan los profesionales? Enferm Intensiva. 2010;21(2):52-7.

16. Burchardi H. Let's open the door! Intensive Care Med. 2002;28(10):1371-2.

17. Kleinpell RM. Visiting hours in the intensive care unit: more evidence that open visitation is beneficial. Crit Care Med. 2008;36(1):334-5.

18. Levy MM. A view from the other side. Crit Care Med. 2007;35(2):603-4.

19. Slota M, Shearn D, Potersnak K, Haas L. Perspectives on familycentered, flexible visitation in the intensive care unit setting. Crit Care Med. 2003;31(5Supp1):S362-6.

20. Simoni R, da Silva M. The impact of the visit of nursing on the necessities of the host families of ICU. Rev Esc Enferm USP.2012;46 Spec No: 65-70. 
21. Roland P, Russell J, Richards K, Sullivan S. Visitation in critical care: processes and outcomes of a performance improvement initiative. J Nurs Care Qual. 2001;15(2):18-26.

22. AACN Practice Alerts. Family presence: visitation in the Adult ICU. Crit Care Nurs. 2012;32(4):76-8.

23. Fumagalli S, Boncinelli L, Lo Nostro NA, Valoti P, Baldereschi G, Di Bari M, et al. Reduced cardiocirculatory complications with unrestrictive visiting policy in an intensive care unit: results from a pilot, randomized trial. Circulation. 2006;113(7):946-52.

24. Bergbom I, Askwall A. The nearest and dearest: a lifeline for ICU patients. Intensive Crit Care Nurs. 2000;16(6):384-95.

25. Happ MB, Swigart VA, Tate JA, Arnold RM, Sereika SM, Hoffmann LA. Family presence and surveillance during weaning from prolonged mechanical ventilation. Heart Lung. 2007;36(1):47-57.

26. Plowright C. Visiting practices in hospitals. Nurs Crit Care. 2007;12(2): 61-3.

27. Ibarrondo B, Tricas S. La visita flexible en las unidades de cuidados intensivos: beneficios para los familiares del paciente crítico. Enferm Intensiva. 2012;23(4):179-88.

28. Garrouste-Org, Philippart F, Timsit JF, et al. Perceptions of a 24-hour visiting policy in the intensive care unit. Crit Care Med. 2008;36(1):30-5.

29. McAdam JL, Arai S, Puntillo KA. Unrecognized contributions of families in the intensive care unit. Intensive Care Med. 2008;34(6):1097101.

30. Gibson V, Plowright C, Collins T, Dawson D, Evans S, Sturmey G, et al. Position statement on visiting in adult critical care units in the UK. Nursi Crit Care. 2012;17(4):213-8.

31. Azoulay E, Pochard F, Kentish-Barnes N, Chevret S, Aboab J, Adrie $\mathrm{C}$, et al. Risk of post-traumatic stress symptoms in family members of intensive care unit patients. Am J Respir Crit Care Med. 2005;171(9):987-94.

32. Williams CMA. The identification of family members' contribution to patients' care in the intensive care unit: a naturalistic inquiry. Nurs Crit Care. 2005;10:6-14.

33. Gonzalez CE, Carroll DL, Elliott JS, Fitzgerald PA, Vallent HJ. Visiting preferences of patients in the intensive care unit and in a complex care medical unit. Am J Crit Care. 2004;13:194-7.

34. Engstrom A, Soderberg S. Critical care nurses' experiences of followup visits to an ICU. J Clin Nurs. 2010;19(19-20):2925-32.

35. Kirchhoff K, Dahl N. American Association of Critical-Care Nurses' National Survey of Facilities and Units Providing Critical Care. Am J Crit Care. 2006;15(1):13-28.

36. Giannini A, Miccinesi G, Leoncino E. Visiting policies in Italian intensive care units: a nationwide survey. Intensive Care Med. 2008;34(7):1256-62. 
37. Quinio P, Savry C, Deghelt A, Guilloux M, Catineau J, de Tinténiac A. A multicenter survey of visiting policies in French intensive care units. Intensive Care Med. 2002;28(10):1389-94.

38. Velasco J, Prieto J, Castillo J, Merino N, Perea-Milla E. Organización de las visitas de familiares en las unidades de cuidados intensivos de España. Enferm Intensiva 2005;16(2):73-83.

39. Spreen A, Schuurmans M. Visiting policies in the adult intensive care units: a complete survey of Dutch ICUs. Intensive Crit Care Nurs. 2011;27(1):27-30.

40. Hunter J, Goddard C, Rothwell M, Ketharaju S, Cooper H. A survey of intensive care unit visiting polices in the United Kindom. Anaesthesia. 2010;65(11):1101-5.

41. Lee MD, Friedenberg AS, Mukpo DH, Conray K, Palmisciano A, Levy MM. Visiting hours policies in New England intensive care units: strategies for improvement. Crit Care Med. 2007;35(2):497-501.

42. Knutsson SE, Otterberg CL, Bergbom IL. Visits of children to patients being cared for in adult ICUs: policies, guidelines and recommendations. Intensive Crit Care Nurs. 2004;20(5):264-74.

43. Haghbin S, Tayebi Z, Abbasian A, and Haghbin H. Visiting hour policies in intensive care units, southern Iran. Iran Red Crescent Med J. 2011 Sep;13(9):684-6.

44. Challan-Belval A, Floccard B, Pereira S, Allaouchiche B. A 24-hour visiting policy in an intensive care unit: Family, patient and nurses' satisfaction. Ann Fr Anesth Réanim. 2013;32(2):123-4.

45. Livesay S, Gilliam A, Mokracek M, Sebastian S, Hickey JV. Nurses' perceptions of open visiting hours in neuroscience intensive care unit. J Nurs Care Qual. 2005;20:182-9.

46. Agard A, Lomborg K. Flexible family visitation in the intensive care unit: nurses' decision-making. J Clin Nurs. 2010;20(7-8):1106-14.

47. Gálvez M, Fernández C, Muñumel G, Rios F, Fernández L Águila B. Acompañamiento familiar: una herramienta para dignificar el processo de muerte en la unidad de cuidados intensivos. Index Enferm. 2011;20(4):233-7.

48. Eriksson T, Lindahl B, Bergbom I. Visits in an intensive care unit-an observational hermeneutic study. Intensive Crit Care Nurs. 2010;26(1):51-7.

49. Zaforteza Lallemand C. Familiares del paciente crítico: sobre las dificultades de la enfermera de la unidad de cuidados intensivos (UCI). Presencia. 2005;1(2).

50. Zaforteza C, Pedro JE, Gastaldo D, Lastra PM, Sánchez-Cuenca P. ¿Qué perspectivas tienen las enfermeras de unidades de cuidados intensivos de su relación con los familiares del paciente crítico? Enferm Intensiva 2003;14(3):109-19. 
